\title{
Caracterización estructural de vidrios con altos contenidos de óxidos de hierro obtenidos a partir de un residuo de la hidrometalurgia del zine ${ }^{(\bullet)}$
}

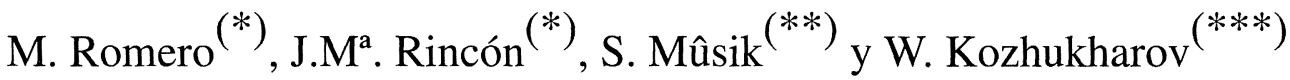 \\ Resumen En este trabajo se ha llevado a cabo la caracterización estructural de vidrios con altos contenidos de \\ óxidos de hierro obtenidos por fusión de un residuo industrial de goethita procedente de la \\ hidrometalurgia del zinc con otras materias primas (dolomita y casco de vidrio). El estudio \\ estructural se ha realizado por difracción de rayos-X (DRX), difracción de rayos-X por dispersión \\ amorfa (RDF) y espectroscopía Mössbauer, determinándose las distancias interatómicas, el estado de \\ oxidación y la coordinación de los átomos de hierro en este nuevo tipo de vidrios.
}

Palabras clave: Estructura de vidrios. Vidrios con altos contenidos de hierro. Goethita. Función de distribución radial. Espectroscopía Mössbauer.

\section{Structural characterization of high iron oxide content glasses obtained from zinc hydrometallurgy wastes}

\begin{abstract}
It has been carried out the structural characterization of high oxide content glasses obtained by melting of a goethite industrial waste from the zinc hydrometallurgy with other raw materials as dolomite and glass cullet. The structural characterization has been carried out by X-Ray Diffraction (XRD), X-Ray Diffraction by Amorphous Dispersion (RDF) and Mössbauer spectroscopy. It has been determined the interatomic distance, the oxidation state and the coordination of iron atoms in these glasses.
\end{abstract}

Keywords: Glasses structure. High iron content glasses. Goethite. Mössbauer spectroscopy. Radial distribution function.

\section{INTRODUCCIÓN}

Los procesos hidrometalúrgicos para la fabricación de zinc metálico se resumen fundamentalmente en dos: a) Proceso de la goethita $(\mathrm{FeOOH})$ y b) Proceso de la jarosita $\left(\mathrm{MFe}_{3}\left(\mathrm{SO}_{4}\right)_{2}(\mathrm{OH})_{6}\right)$. En ambos procesos se produce una elevada cantidad de residuos en forma de fangos o lodos (600.000 t/año en

(•) Trabajo recibido el día 21 de enero de 1997.

(*) Lab. de Materiales Vitrocerámicos. Instituto E. Torroja de Ciencias de la Construcción (CSIC). C/ Serrano Galvache, s/n 28033-Madrid (España).

(**) Ruder Boskovic Institute. P.O. Box 1016. 10001 Zagreb (Croacia)

${ }^{(* * *)}$ Higher Institute of Technology. University of Sofia. K1. Okhridski, 1756 Sofia (Bulgaria). los países de la Unión Europea) que hasta ahora permanecen almacenados en depósitos especiales próximos a las fábricas. La actual saturación de estos depósitos constituye hoy en día un gran problema medioambiental y económico a nivel mundial.

En los últimos años se están realizando varios trabajos de investigación con el fin de reciclar estos residuos, ricos en óxidos de hierro y metales pesados, a través de su fusión con materias primas adecuadas para obtener vidrios y materiales vitrocerámicos con elevados contenidos de óxido de hierro, estables al medio ambiente (1) y que pueden ser utilizados como materiales de construcción.

Con el objetivo de conocer la relación entre estructura, microestructura y propiedades de vidrios y materiales vitrocerámicos con elevados contenidos de óxido de hierro que pueden ser obtenidos a partir de una amplia gama de residuos industriales, en este 
trabajo se ha llevado a cabo la caracterización estructural por medio de técnicas de difracción de rayos-X (DRX), difracción de rayos-X por dispersión amorfa (RDF) y espectroscopía Mössbauer de tres vidrios (G1, G5 y G9) que forman parte de una serie de once vidrios obtenidos por la fusión de mezclas de residuos de goethita con dolomita y casco de vidrio (2). Se han elegido los vidrios G1, G9 y G5 por ser los que tienen mayor, menor e intermedio contenido de óxidos de hierro respectivamente.

\section{PROCEDIMIENTO EXPERIMENTAL}

La tabla I muestra la composición química de los vidrios determinada por el método de acoplamiento de plasma inductivo. La fusión se ha llevado a cabo en crisoles sílico-aluminosos a $1.450{ }^{\circ} \mathrm{C}$ durante $30 \mathrm{~min}$, seguida de un recocido a $500{ }^{\circ} \mathrm{C}$ durante $2 \mathrm{~h}$.

La naturaleza amorfa de los vidrios ha sido estudiada en un difractómetro Siemens D5000 con radiación $\mathrm{K}_{\alpha}$ de cobre sobre muestras molidas hasta su paso por tamiz de $37 \mu \mathrm{m}$.

El estudio de difracción de rayos-X por dispersión amorfa se ha realizado en un equipo URD6 semiautomático (compatible con Siemens) con radiación $\mathrm{K}_{\alpha}$ de molibdeno. El tratamiento de datos, cálculo de los factores estructurales y función de distribución radial se ha realizado con el programa RAD (3).

Esta técnica se basa en que la intensidad de la radiación difractada por un grupo de átomos orientados en todas las direcciones del espacio se obtienen, en función del ángulo de difracción, mediante la ecuación de Debye:

$$
I=\Sigma_{\mathrm{m}} \Sigma_{\mathrm{n}} f_{\mathrm{m}} f_{\mathrm{n}} \frac{\operatorname{sen}\left(s r_{\mathrm{mn}}\right)}{s r_{\mathrm{mn}}}
$$

TABLA I.- Composición química en óxidos de los vidrios investigados, $\%$ en peso

TABLE I.- Chemical composition of glasses investigated, wt \%

\begin{tabular}{|l|r|r|r|}
\hline & \multicolumn{1}{|c|}{$\mathrm{G} 1$} & \multicolumn{1}{c|}{$\mathrm{G} 5$} & \multicolumn{1}{c|}{$\mathrm{G} 9$} \\
\hline $\mathrm{SiO}_{2}$ & 38,41 & 42,68 & 44,90 \\
$\mathrm{Al}_{2} \mathrm{O}_{3}$ & 6,42 & 8,04 & 3,63 \\
$\mathrm{Fe}_{2} \mathrm{O}_{3}$ & 29,24 & 20,53 & 18,04 \\
$\mathrm{CaO}$ & 6,63 & 11,24 & 13,99 \\
$\mathrm{MgO}$ & 2,85 & 5,37 & 6,58 \\
$\mathrm{ZnO}$ & 9,47 & 6,15 & 5,27 \\
$\mathrm{PbO}$ & 2,24 & 1,52 & 1,32 \\
$\mathrm{Na} 2 \mathrm{O}$ & 3,92 & 3,61 & 5,68 \\
$\mathrm{~K}_{2} \mathrm{O}$ & 0,81 & 0,86 & 0,60 \\
\hline
\end{tabular}

donde $f_{\mathrm{m}}$ y $f_{\mathrm{n}}$ representan los valores de difracción para los átomos $m$ y $n ; r_{\mathrm{mn}}$ es la distancia entre dichos átomos. Si tales distancias son conocidas, la intensidad se obtiene de un modo inmediato en función del ángulo, ya que $s$ es función del ángulo $\theta$ y de la longitud de onda $\lambda$ de la radiación empleada:

$$
s=\frac{4 \pi \operatorname{sen} \theta}{\lambda}
$$

Warren y col. (4) realizando un análisis de Fourier de la curva experimental de difracción de rayos-X obtuvieron la función de distribución radial (RDF) que representa la densidad atómica o número de átomos que rodean a un átomo de referencia en función de su distancia al mismo. A partir de las curvas de distribución radial, se puede obtener información, tanto del orden de corto alcance (SRO) como del orden de largo alcance (LRO) de la estructura vítrea. En el caso de una sustancia que presente un completo desorden estructural, su densidad electrónica radial aumentará con la distancia siguiendo una función parabólica. Si por el contrario, la curva se desvía de su curso parabólico, se pone de manifiesto un cierto grado de ordenación. Así pues, a partir de las posiciones de los máximos de los picos se pueden conocer las distancias al átomo de referencia, mientras que las áreas delimitadas por ellos son proporcionales al número de átomos que le rodean.

La espectroscopía Mössbauer es una técnica de resonancia nuclear que utilizando el fenómeno descubierto por Mössbauer en 1958 (5) estudia las transiciones entre los diferentes niveles de energía de un material sólido por efecto de la radiación $\gamma$ $(14,4 \mathrm{keV})$ que es emitida o absorbida por un material sin pérdida de energía de retroceso. Cuando hay pérdidas de energía por retroceso de los átomos emisor y absorbente se destruye la resonancia; para restablecerla se varía la energía del sistema emisorabsorbente por efecto Doppler. Por tanto, un espectro Mössbauer es la representación de la absorción relativa de la radiación en función de la velocidad relativa entre la fuente y el absorbente. Básicamente, el método permite observar pequeñas interacciones entre los núcleos resonantes (en este caso $\mathrm{Fe}^{57}$ ) y su entorno electrónico.

Los parámetros fundamentales que se obtienen de un espectro Mössbauer son: el desplazamiento isomérico $(\delta)$, el desdoblamiento de cuadrupolo $(\Delta)$ y la interacción magnética hiperfina. El desplazamiento isomérico es el resultado de la diferente densidad electrónica nuclear de la fuente y el absorbente. En el caso del $\mathrm{Fe}^{57}$, el desplazamiento isomérico depende del estado de oxidación del hierro, de la naturaleza de los aniones coordinados y del grado de 
covalencia, mientras que el desplazamiento de cuadrupolo depende de la simetría de cargas del entorno atómico debida a los ligandos coordinados alrededor del hierro. Por último, la interacción magnética hiperfina es el resultado de la interacción entre el momento magnético del núcleo y el campo magnético externo. La espectroscopía Mössbauer ha sido aplicada ampliamente al estudio del estado de oxidación del hierro en vidrios, así como a la determinación de la relación $\mathrm{Fe}^{3+} / \mathrm{Fe}^{2+}$ (6 y 7).

\section{RESULTADOS Y DISCUSIÓN}

\subsection{Difracción de rayos-X (DRX)}

La figura 1 muestra los difractogramas de rayos$\mathrm{X}$ de los vidrios aquí investigados observándose notables diferencias entre ellos. Así, los vidrios G5 y G9 muestran un halo amorfo por difracción de rayos-X mientras que el difractograma correspondiente a la composición G1 presenta picos bien definidos que corresponden a la cristalización de magnetita. Por microscopía electrónica se ha determinado que la fracción de volumen de la fase magnetita es del $80 \%$. Los cristales de magnetita se encuentran formando una red dendrítica con un tamaño medio de cristal de $0,7 \mu \mathrm{m}$.

\subsection{Difracción de rayos-X por dispersión amorfa (RDF)}

En los vidrios estudiados se ha determinado el orden de corto alcance (SRO) a través de la Función de Distribución Radial (RDF) que, como ya se ha visto anteriormente, es una función del factor de

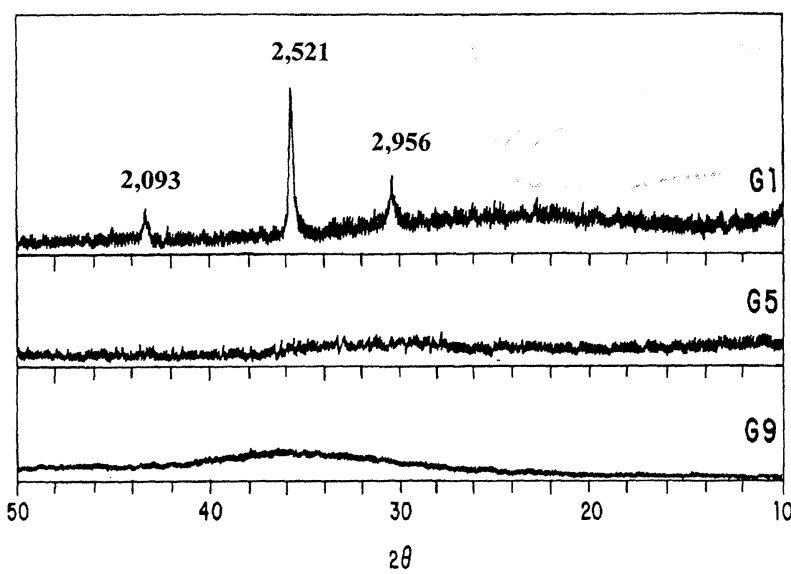

FIG. 1.- Difractogramas de rayos-X de los vidrios investigados.

FIG. 1.-X-Ray diffractograms for glasses here investigated. estructura de la red amorfa, que a su vez es función de:

- La dispersión de Bragg, $2 \pi \operatorname{sen} \theta / \lambda$

- El sumatorio de distancias interatómicas por el factor de absorción atómica para cada uno de los elementos atómicos implicados en la dispersión de los rayos $\mathrm{X}$.

Así pues, en estos vidrios que básicamente pertenecen al sistema de composición $\mathrm{ZnO}-\mathrm{CaO}$ $\mathrm{Fe}_{2} \mathrm{O}_{3}-\mathrm{Al}_{2} \mathrm{O}_{3}-\mathrm{SiO}_{2}$, la función de distribución radial dará información de los enlaces $\mathrm{Si}-\mathrm{O}, \mathrm{O}-\mathrm{O}, \mathrm{Si}-\mathrm{Ca}$, $\mathrm{Si}-\mathrm{Zn}, \mathrm{Al}-\mathrm{O}, \mathrm{Fe}-\mathrm{O}$, etc.

La figura 2 representa las gráficas de intensidad de rayos X frente a la dispersión angular. Se observa una fuerte dispersión en el intervalo $2 \theta=10$ $40^{\circ}$ (orden de corto alcance) habiendo una disminución progresiva de la dispersión de los rayos-X desde 40 hasta $80^{\circ}$ (orden de largo alcance). A partir de estos difractogramas, se ha obtenido la representación de la función de interferencia frente a la función de dispersión del ángulo de Bragg $(s)$ (Fig. 3 ). Como a partir de estas gráficas no es posible obtener directamente información estructural, se ha aplicado la transformada de Fourier para así obtener la función de distribución radial de la densidad electrónica, $g(r)$, como se muestra en la figura 4. En cualquier caso, existe más semejanza entre los vidrios G5 y G9, mostrando el material G1 mayor orden de largo alcance, debido a la cristalización de magnetita como ya se ha observado anteriormente en el análisis difractométrico.

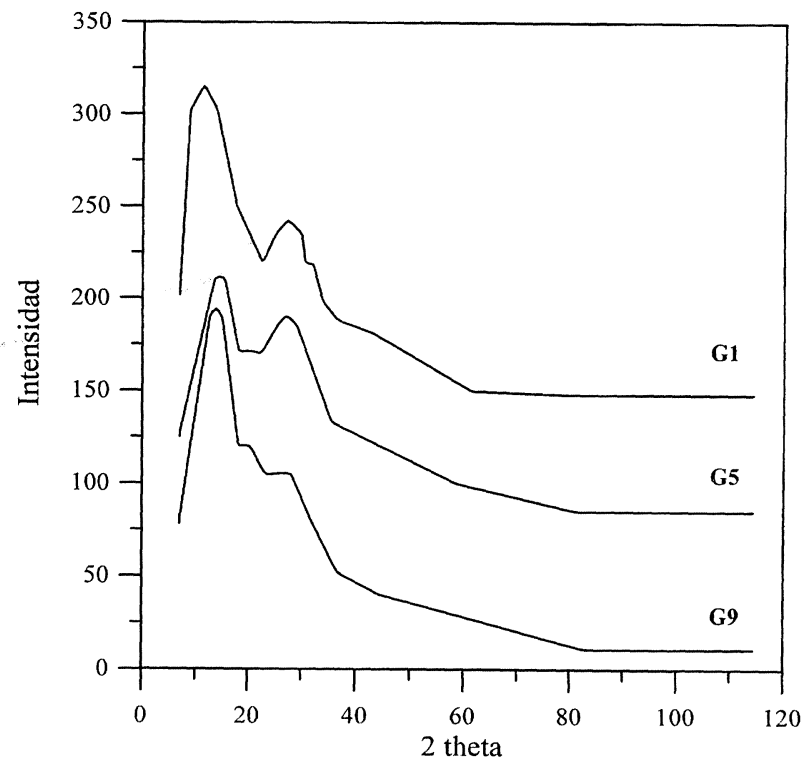

FIG. 2.- Representación de intensidad frente a dispersión angular para los vidrios G1, G5 y G9.

FIG. 2.- Intensity versus angular dispersion for G1, G5 and G9 glasses. 


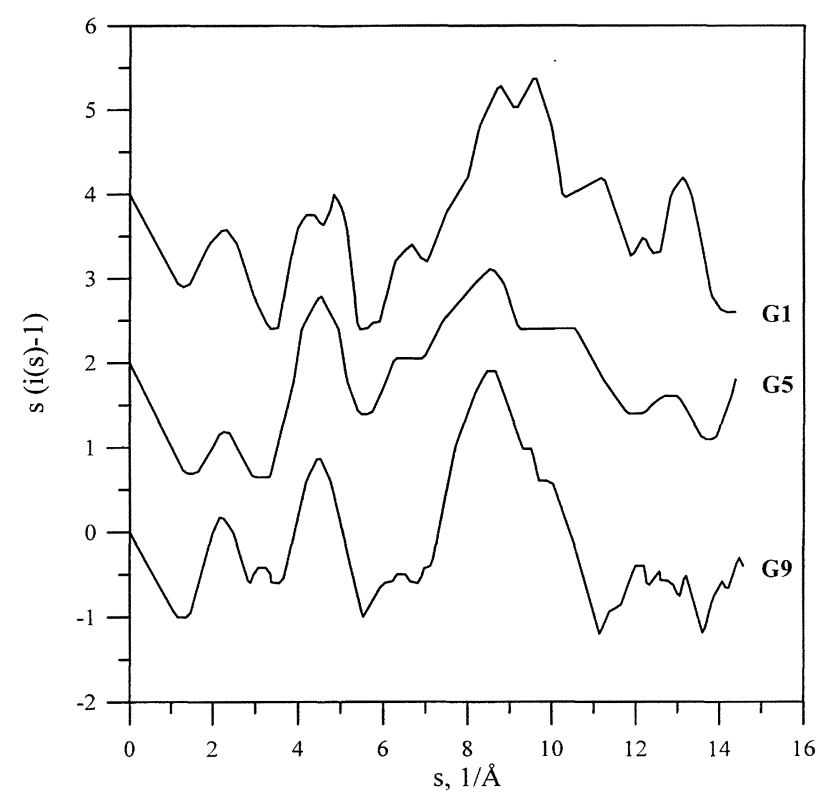

FIG. 3.- Representación de la función de interferencia frente a la función de dispersión del ángulo de Bragg ( $s$ ) para los vidrios G1, G5 y G9.

FIG. 3.- Interference function versus Bragg angle dispersion function (s) for G1, G5 and G9 glasses.

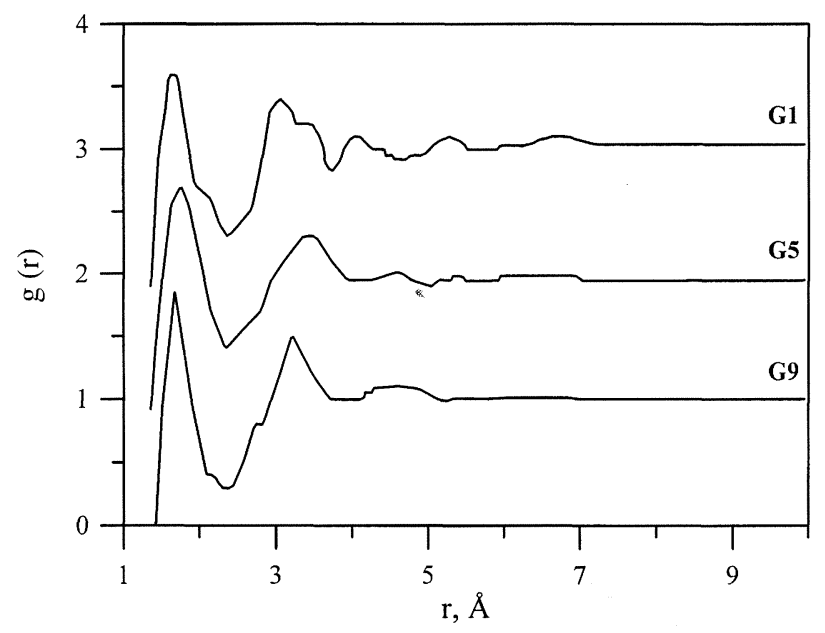

FIG. 4.- Función de distribución radial (RDF) de la densidad electrónica, $g(r)$, para los vidrios G1, G5 y G9.

FIG. 4.- Radial Distribution Function (RDF) of electronic density from $G 1, G 5$ and $G 9$ glasses.

De acuerdo con los trabajos previos de Warren y col. (4) y los datos expresados en Fernández-Navarro (8), en la tabla II se dan las distancias interatómicas teóricas calculadas a partir de los radios iónicos, así como las obtenidas experimentalmente a partir de la figura 4.

El vidrio G1, que es el que presenta mayor orden de largo alcance, muestra también una mayor
TABLA II.- Distancias interatómicas teóricas y experimentales para los vidrios G1, G5 y G9

TABLE II.- Theoretic and experimental interatomic distance from $G 1, G 5$ and $G 9$ glasses

\begin{tabular}{|c|c|c|c|c|}
\hline & \multicolumn{4}{|c|}{$\mathrm{r}(\AA)$} \\
\hline & \multirow{2}{*}{ Teórico } & \multicolumn{3}{|c|}{ Experimental } \\
\hline & & G1 & G5 & G9 \\
\hline $\mathrm{Si}-\mathrm{O}$ & 1,60 & 1,60 & 1,70 & 1,68 \\
\hline $\mathrm{O}-\mathrm{O}$ & 2,60 & - & - & 2,73 \\
\hline $\mathrm{Si}-\mathrm{Si}$ & 3,20 & 3,00 & 3,15 & 3,20 \\
\hline $\mathrm{Al}-\mathrm{O}$ & 1,88 & 1,85 & - & - \\
\hline $\mathrm{Fe}^{3+-} \mathrm{O}(\mathrm{T})$ & 1,84 & 1,85 & - & - \\
\hline $\mathrm{Fe}^{3+-} \mathrm{O}(\mathrm{O})$ & 1,99 & 1,85 & - & - \\
\hline $\mathrm{Fe}^{2+-} \mathrm{O}(\mathrm{T})$ & 2,12 & 1,85 & - & 2,10 \\
\hline $\mathrm{Zn}-\mathrm{O}$ & 2,10 & - & 4,30 & 4,30 \\
\hline $\mathrm{Pb}-\mathrm{O}$ & 2,12 & & & \\
\hline
\end{tabular}

complejidad en el orden de corto alcance. Se identifican claramente los picos debidos a los enlaces Si$\mathrm{O}, \mathrm{Si}-\mathrm{Si}$; sin embargo, hay un solapamiento de los picos correspondientes a los enlaces $\mathrm{Al}-\mathrm{O}$ y Fe-O (para $\mathrm{Fe}^{3+}$, octaédrico y tetraédrico y $\mathrm{Fe}^{2+}$ octaédrico) por lo que no ha sido posible la determinación de estas distancias interatómicas.

Respecto a los vidrios G5 y G9, su espectro RDF es más sencillo, identificándose únicamente de manera clara las distancias Si-O y Si-Si. Respecto al pico que aparece en $4,30 \AA$ debe corresponder al enlace $\mathrm{Fe}-\mathrm{O}$ (para $\mathrm{Fe}^{2+}$ en coordinación octaédrica) o a enlaces $\mathrm{Zn}-\mathrm{O}$ ó Pb-O en coordinación octaédrica y puede estar asociado al pico correspondiente a la distancia $\mathrm{O}-\mathrm{O}$ (no puente) que se ve claramente en el vidrio G9 y se intuye en el G5.

Por otro lado, se han observado desplazamientos en las distancias interatómicas debidos a la dificultad de acoplamiento de todos los elementos diferentes en la red vítrea, ya que se trata de vidrios de ocho componentes. Además, algunos de estos iones modificadores, como es el caso del $\mathrm{Pb}^{2+}$, son iones con muy alta polarizabilidad que deformarían la red de silicato de manera análoga a como se ha comprobado también por determinaciones de RDF en vidrios con altas cantidades de $\mathrm{Cd}^{2+}(9)$.

\subsection{Espectroscopía Mössbauer}

La figura 5 muestra los espectros Mössbauer obtenidos para el material petrúrgico G1 y para los vidrios G5 y G9; estos últimos presentan un espectro sin componentes magnéticos con bandas de 

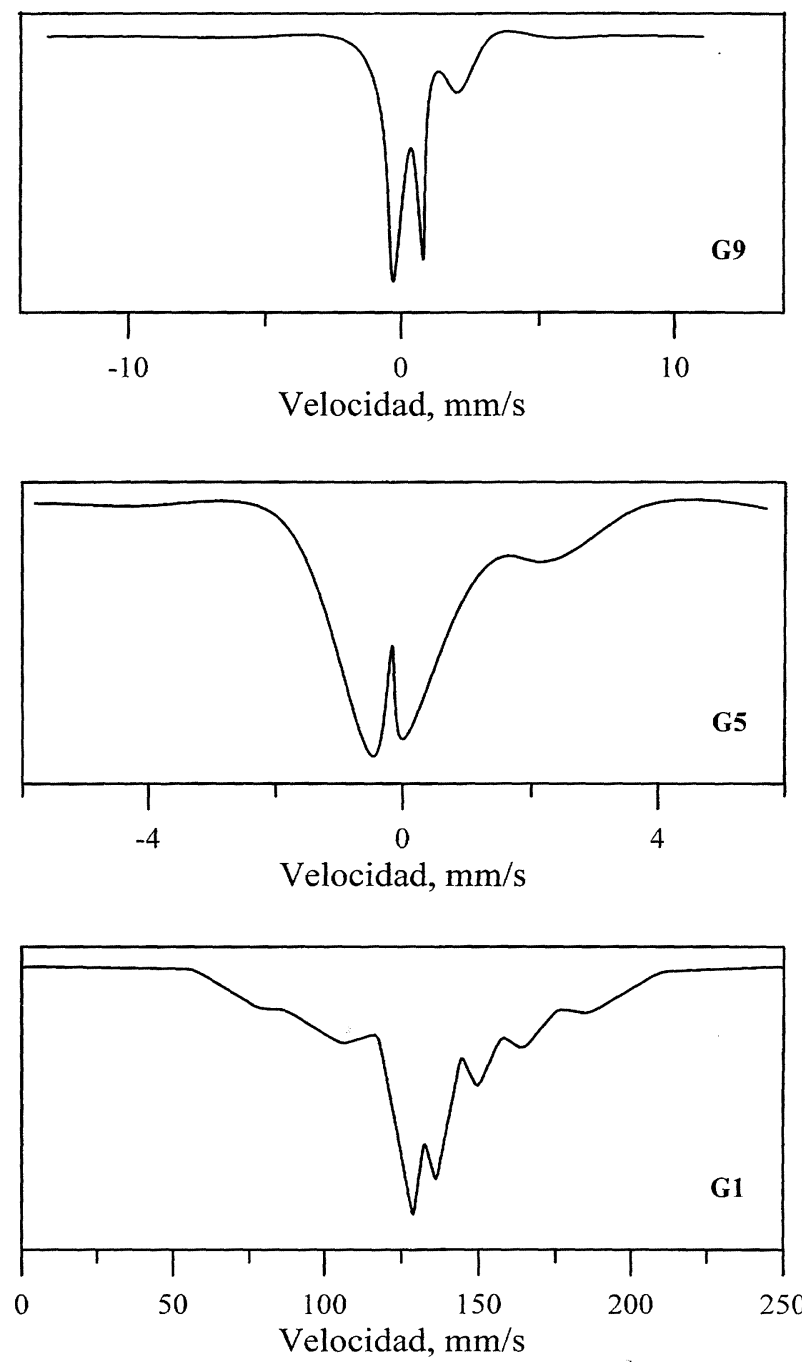

FIG. 5.- Espectros Mösbauer obtenidos para el material petrúrgico G1 y para los vidrios G5 y G9.

FIG. 5.- Mössbauer spectra obtained for the petrurgic $G 1$ and for $G 5$ and $G 9$ glasses.

absorción en la parte central del espectro. En el material petrúrgico G1, junto a estas bandas aparecen, como era de esperar, una componente magnética típica de la magnetita. Un ajuste matemático de estos espectros permite la deconvolución de las bandas, dando lugar a una serie de parámetros Mössbauer recogidos en la tabla III.

Una vez realizada la deconvolución, los valores de los parámetros Mössbauer de cada uno de los dobletes o sextetes permite conocer el estado de oxidación y la coordinación de los átomos de hierro asociados a ellos (10). De igual forma, el área de cada uno de los picos deconvolucionados permite conocer el porcentaje de hierro en cada uno de los estados. La tabla IV recoge los resultados obtenidos para las composiciones ensayadas. Representando los valores del desplazamiento isomérico $(\delta)$ frente al desdoblamiento de cuadrupolo $(\Delta)$, de acuerdo
TABLA III.- Parámetros Mössbauer obtenidos para el material petrúrgico G1 y para los vidrios G5 y G9

TABLE III.-Mössbauer parameters for the petrurgic $G 1$ and for $G 5$ and $G 9$ glasses

\begin{tabular}{|c|l|c|c|c|c|}
\hline & \multicolumn{1}{|c|}{ Deconvolución } & & $\delta, \mathrm{mm} / \mathrm{s}$ & $\Delta, \mathrm{mm} / \mathrm{s}$ & $\mathrm{H}, \mathrm{kG}$ \\
\hline \multirow{4}{*}{ G1 } & \multirow{2}{*}{ 2 sextetes + 2 dobletes } & S1 & 0,28 & 0,05 & 438 \\
& & S2 & 0,50 & 0,07 & 381 \\
& & D1 & 1,09 & 2,34 & - \\
& & D2 & 0,49 & 1,67 & - \\
\hline \multirow{4}{*}{ G5 } & \multirow{2}{*}{4 dobletes } & D1 & 0,15 & 1,51 & - \\
& & D2 & 0,99 & 2,12 & - \\
& & D3 & 0,83 & 1,51 & - \\
\hline \multirow{2}{*}{ G9 } & \multirow{2}{*}{ 2 Dobletes } & D4 & 0,15 & 0,92 & - \\
& & D1 & 0,16 & 1,09 & - \\
\hline
\end{tabular}

TABLA IV.- Estado de oxidación, coordinación y porcentaje de hierro en cada uno de los estados obtenidos a partir de los valores de los parámetros Mössbauer para el material petrúrgico G1 y para los vidrios G5 y G9

TABLE IV.- Oxidation state, coordination and iron content for each one of states obtained from Mössbauer parameters for the petrurgic Gl and for G5 and $G 9$ glasses

\begin{tabular}{|c|c|c|c|c|c|}
\hline & $\begin{array}{c}\text { Decon- } \\
\text { volución }\end{array}$ & $\begin{array}{c}\text { Estado de } \\
\text { oxidación }\end{array}$ & $\begin{array}{c}\text { Coordi- } \\
\text { nación }\end{array}$ & $\%$ & $\mathrm{Fe}^{3+} / \mathrm{Fe}^{2+}$ \\
\hline \multirow{4}{*}{ G1 } & $\mathrm{S} 1$ & $\mathrm{Fe}_{3} \mathrm{O}_{4}$ & - & 17,0 & \\
& $\mathrm{~S} 2$ & $\mathrm{~F}_{3} \mathrm{O}_{4}$ & - & 41,4 & 2,6 \\
& D1 & $\mathrm{Fe}^{2+}$ & octaédrica & 11,4 & \\
& D2 & $\mathrm{Fe}^{3+}$ & tetraédrica & 30,2 & \\
\hline \multirow{4}{*}{ G5 } & D1 & $\mathrm{Fe}^{3+}$ & tetraédrica & 28,0 & \\
& D2 & $\mathrm{Fe}^{2+}$ & octaédrica & 12,6 & 4,9 \\
& D3 & $\mathrm{Fe}^{2+}$ & - & 4,1 & \\
& D4 & $\mathrm{Fe}^{3+}$ & tetraédrica & 55,3 & \\
\hline \multirow{2}{*}{ G9 } & D1 & $\mathrm{Fe}^{3+}$ & tetraédrica & 84,3 & 5,3 \\
& D2 & $\mathrm{Fe}^{2+}$ & octaédrica & 15,7 & \\
\hline
\end{tabular}

con Dyar (11), se tiene una idea monográfica de la situación de las distintas posiciones frente a la que tiene lugar en vidrios semejantes de silicato que contienen óxido de hierro (Fig. 6).

El hierro en este tipo de vidrios se encuentra en la red vítrea en dos estados de oxidación: $\mathrm{Fe}^{2+}$ y $\mathrm{Fe}^{3+}$. Junto a estos, en el material petrúrgico G1 aparecen dos sextetes típicos de la magnetita con valores de campo magnético hiperfino de $438 \mathrm{kG}$ y $381 \mathrm{kG}$, encontrándose la mayoría del hierro en esta fase $(58,4 \%)$. Si se mira la relación $\mathrm{Fe}^{3+} / \mathrm{Fe}^{2+}$, ésta varía de unos vidrios a otros, pasando de un valor de 2,6, para el material petrúrgico $\mathrm{G} 1$, hasta un 


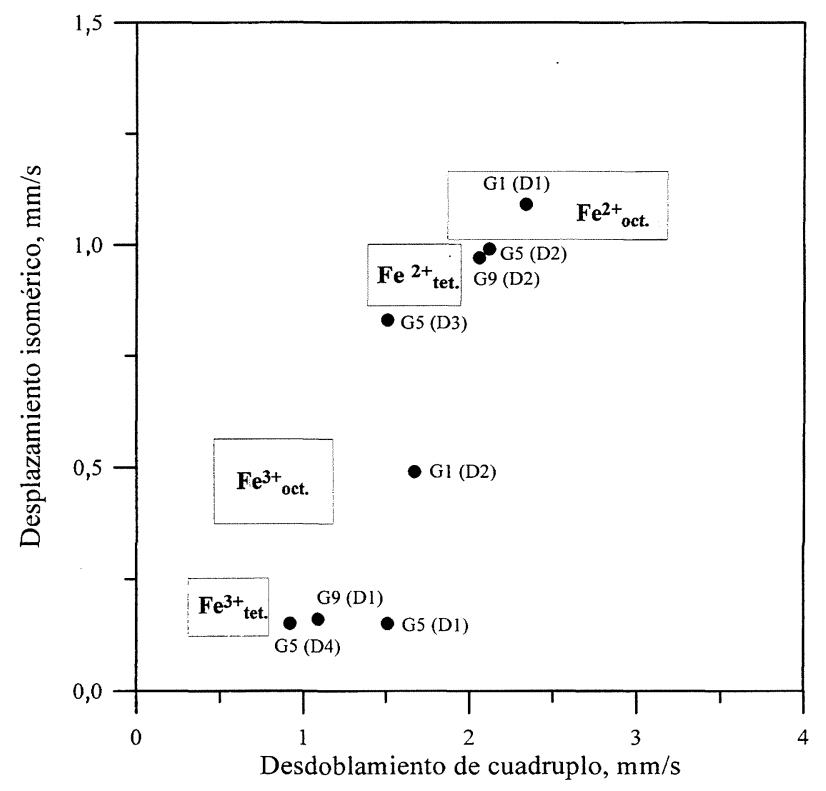

FIG. 6.- Representación del desdoblamiento de cuadrupolo frente al desplazamiento isomérico mostrando las regiones donde según Dyar están situados los diferentes iones hierro en función de sus estados de oxidación y coordinación en vidrios semejantes de silicato conteniendo óxidos de hierro.

FIG. 6.- Quadrupole splitting versus isomer shift showing the different Dyar regions where are located the different iron ions according to their oxidation state and coordination for similar iron oxide silicate glasses.

valor de 1,3 para el vidrio G9. Sobre esta relación $\mathrm{Fe}^{3+} / \mathrm{Fe}^{2+}$ influye una serie de variables como son: la composición del vidrio, la temperatura de fusión, la velocidad de enfriamiento, la atmósfera del horno, etc. Puesto que todas las composiciones han sido fundidas en las mismas condiciones, cabe pensar que es la variación en la composición del vidrio de partida la que origina las diferencias en el valor de la relación $\mathrm{Fe}^{3+} / \mathrm{Fe}^{2+}$. En este caso, aunque el contenido total en óxido de hierro disminuye de G1 a G9, estas diferencias son difíciles de explicar, puesto que al pasar de una composición a otra se producen variaciones no secuenciales en el porcentaje de todos los óxidos mayoritarios del vidrio (12).

Asimismo, dado que la conductividad térmica de cada uno de los vidrios es diferente, debido al distinto contenido de óxidos de hierro, la variación en la disipación de calor en el enfriamiento puede afectar también a la relación final $\mathrm{Fe}^{3+} / \mathrm{Fe}^{2+}$. En cuanto a la coordinación del hierro en estos vidrios, en todos los casos el $\mathrm{Fe}^{3+}$ en la red vítrea adopta coordinación tetraédrica actuando como formador de red. Este hecho está de acuerdo con resultados obtenidos por otros autores (13 y 14) que encuen- tran que la coordinación tetraédrica es la ocupada por todos los iones férricos cuando, como en este caso, la relación $\mathrm{Fe}^{3+} / \mathrm{Fe}^{2+}>1$. En G1 y G9 todos los iones $\mathrm{Fe}^{2+}$ se encuentran en coordinación octaédrica (15). En el vidrio G5, los iones $\mathrm{Fe}^{2+}$ se encuentran mayoritariamente en coordinación octaédrica, pero se observa una nueva posición (correspondiente al doblete D3) cuyos valores de desdoblamiento isomérico y desplazamiento de cuadrupolo están más cercanos a $\mathrm{Fe}^{2+}$ con coordinación tetraédrica $(\delta=0,9-1 \mathrm{~mm} / \mathrm{s}$ y $\Delta=1,4-2$ $\mathrm{mm} / \mathrm{s}$ ). Es preciso señalar que no existen datos sobre este tipo de coordinación para los cationes $\mathrm{Fe}^{2+}$ en vidrios de silicato fundidos en condiciones oxidantes, asociándose en algunos casos a posiciones octaédricas deformadas (16).

Se observa también que el porcentaje de $\mathrm{Fe}^{2+}$ en esta posición es muy bajo $(4,1 \%)$ no apareciendo en los otros materiales G1 y G9, debido posiblemente al balance de oxígeno puente y no-puente de este vidrio intermedio en el que se pasa de contenidos altos a contenidos más bajos de óxido de hierro. En el material petrúrgico $\mathrm{G} 1$, los iones $\mathrm{Fe}^{3+}$ se encuentran en la fase vítrea ocupando una única posición tetraédrica (P1) que recoge al $100 \%$ de la población de iones $\mathrm{Fe}^{3+}$. Cuando pasamos al vidrio G5, junto a esta posición tetraédrica aparece otra con igual valor de desplazamiento isomérico $(\delta=$ $0,15 \mathrm{~mm} / \mathrm{s}$ ) pero diferente valor de desdoblamiento de cuadrupolo. La posición correspondiente al doblete D1 con $\Delta=1,51 \mathrm{~mm} / \mathrm{s}$, que sería semejante a la posición tetraédrica $\mathrm{P} 1$ en el material petrúrgico G1, contiene en este caso sólo un $33,6 \%$ de la población total de iones $\mathrm{Fe}^{3+}$, mientras que el 66,4 $\%$ restante ocupa una posición tetraédrica diferente (P2), correspondiente al doblete D4 con menor valor de desdoblamiento de cuadrupolo, $\Delta=0,92$ $\mathrm{mm} / \mathrm{s}$. Esta diferencia en el valor del desdoblamiento de cuadrupolo indica una variación en la distorsión del entorno que rodea a los iones $\mathrm{Fe}^{3+}$. Así, la posición tetraédrica $\mathrm{P} 2$ con menor valor de desdoblamiento de cuadrupolo tendrá menor distorsión que la posición tetraédrica P1. En el vidrio G9, el $100 \%$ de los iones $\mathrm{Fe}^{3+}$ ha pasado a ocupar la posición menos distorsionada $\mathrm{P} 2$.

En cuanto a los iones $\mathrm{Fe}^{2+}$, estos ocupan preferentemente posiciones octaédricas y al igual de lo que ocurría con los iones $\mathrm{Fe}^{3+}$, se puede observar que según disminuye el contenido de óxido de hierro, las posiciones octaédricas son más simétricas, pasando de un valor de $\Delta=2,34 \mathrm{~mm} / \mathrm{s}$ en el material petrúrgico $\mathrm{G} 1$ a una posición menos distorsionada con $\Delta=2,06 \mathrm{~mm} / \mathrm{s}$ en el vidrio G9. En el material petrúrgico $\mathrm{G} 1$, la totalidad de los iones ferrosos se encuentran en una única posición octaédrica. Al pasar al vidrio G5 con menor contenido de óxido de hierro, esta posición gana simetría 
pasando de $\Delta=2,34 \mathrm{~mm} / \mathrm{s}$ a $\Delta=2,12 \mathrm{~mm} / \mathrm{s}$ y recogiendo un $75 \%$ de la población total de iones $\mathrm{Fe}^{2+}$, encontrándose el $25 \%$ de iones ferrosos restantes en una posición que, como ya se ha comentado anteriormente, puede ser tetraédrica u octaédrica deformada con $\Delta=1,51 \mathrm{~mm} / \mathrm{s}$. Cuando se pasa al vidrio G9 con un contenido de óxido de hierro menor que en los vidrios anteriores, los iones $\mathrm{Fe}^{2+}$ vuelven a encontrarse en una única posición octaédrica que de nuevo ha ganado simetría con un valor de desdoblamiento de cuadrupolo $\Delta=2,06 \mathrm{~mm} / \mathrm{s}$.

\section{CONCLUSIONES}

Se ha realizado la caracterización estructural de vidrios obtenidos por primera vez a partir de mezclas de un residuo de goethita con dolomita y casco de vidrio. Los ensayos de difracción de rayos-X muestran que en la composición G1 ha precipitado cristales de magnetita mientra que los vidrios G5 y G9 son totalmente amorfos.

La función de distribución radial (RDF) de estos vidrios con alto contenido de óxidos de hierro muestra un espectro sencillo que indica una red con orden de corto alcance (SRO) en los vidrios con menores contenidos de óxidos de hierro (G5 y G9). Sin embargo, en el material petrúrgico G1 aumenta el orden de largo alcance (LRO), siendo muy complejo el espectro en distancias interatómicas menores de los 0,4 $\mathrm{nm}$. Se han observado desplazamientos en las distancias interatómicas obtenidas a partir de la RDF, debido a la dificultad de acoplamiento en la red vítrea de silicato de los diferentes cationes en estos vidrios multicomponentes.

Por espectroscopía Mössbauer se ha determinado que el hierro se encuentra en la red vítrea en dos estados de oxidación: $\mathrm{Fe}^{+2}$ y $\mathrm{Fe}^{+3}$, con diferentes números de coordinación: octaédrica para el catión ferroso, tetraédrica para el catión férrico en el vidrio G5 y G9, y octaédrica en el caso del G1 que es el que contiene mayor proporción de óxidos de hierro. La relación $\mathrm{Fe}^{+3} / \mathrm{Fe}^{+2}$ determinada por espectroscopía Mössbauer aumenta cuando disminuye el contenido de hierro total del vidrio original.

\section{Agradecimiento}

Los autores agradecen a la UE (Proyecto Europeo Raw Materials-DG XII (MA 2R CT90-0007)) el soporte financiero de este trabajo.

\section{REFERENCIAS}

(1) Romero, M. Procesado y caracterización de nuevos vidrios y materiales vitrocerámicos obtenidos por reciclado de residuos industriales de goethita. Tesis doctoral, Universidad de Alcalá de Henares, Facultad de Ciencias Químicas, 1995.

(2) Romero, M. y Rincon, J. J. Eur. Ceram. Soc. 5, 1997:

(3) Petkov, V. J. Appl. Cryst., 22, 1989: 387-389.

(4) Warren, B.E., Krutter, H. y Morningstar, O. J. Amer. Ceram. Soc., 19 (7), 1936: 202-206.

(5) Stevens, J.G. Handbook of Mineral Data Mössbauer. Ed. Effect Data Center, University of North Carolina, 1983.

(6) KurkJian, C.R. J. Non-Cryst. Solids, 3, 1970: 157-194.

(7) Levy, R.A., LuPis, C.H.P y Flinn, P.A. Phys. Chem. Glasses, 17 (4) 1976: 94-103.

(8) Fernández Navarro, J.M. El Vidrio. Ed. Consejo Superior de Investigaciones Científicas ( $2^{\mathrm{a}}$ ed.), Madrid, 1991.

(9) ZayAs SAUCEDO, $M^{a}$.E. Nuevos vidrios en aplicaciones ópticas formulados en el sistema $\mathrm{ZnO}-\mathrm{CdO}-\mathrm{Al}_{2} \mathrm{O}_{3}-\mathrm{TeO}_{2}$ $\mathrm{SiO}_{2}$. Tesis doctoral. Dpto. de Física de la Materia Condensada, Universidad Autónoma de Madrid, 1994.

(10) TomandL, G. Mössbauer effect in glasses. Glass: Science and Technology. Vol. 4B. Ed. D.R. Uhlmann, N.J. Kreidl, Academic Press, Nueva York, 1990.

(11) Dyar, D.M. J. Am. Ceram. Soc., 69 (7), C160-C162. 1986.

(12) Stevens, J.G. MRS Bull., nov.-dic., 1986: 14-17.

(13) Mysen, B.O., Virgo, D., Neumann, E.R. y Seifert, F.A. Am. Mineral., 70, 1985: 317.

(14) Dickenson, M.P. y Hess, P.C. Contrib. Mineral. Petrol., 92, 1986: 207

(15) Dyar, M.D. Am. Mineral., 70, 1985: 304.

(16) Gancedo, J.R., Gracia, M., Callejas, P. y Rincón, J.Ma. Estudio de la superficie de materiales vitrocerámicos por espectroscopía Mössbauer. XX Reunión Bienal de la Sociedad Española de Física y Química, Castellón, 1984. 